\title{
Photoelectron Resonance Capture Ionization (PERCI): A Novel Technique for the Soft-Ionization of Organic Compounds
}

\author{
Brian W. LaFranchi and Giuseppe A. Petrucci \\ Department of Chemistry, University of Vermont, Burlington, Vermont, USA
}

\begin{abstract}
Photoelectron resonance capture ionization (PERCI) is demonstrated as a sensitive ionization technique involving minimal fragmentation of organic molecules. PERCI has been used successfully to softly and efficiently ionize both strongly UV absorbing and non-absorbing molecules. Tunable low energy $(<1 \mathrm{eV})$ electrons are generated by focusing a pulsed UV laser on an aluminum photocathode in the presence of gas phase analyte. Negative ions are formed through a resonance electron capture process. Mass analysis is done using a reflectron time-of-flight mass spectrometer. PERCI is demonstrated for a number of gas phase compounds and simple mixtures, including sulfur hexafluoride, nitrobenzene, nitrophenol, 2-pentanone, hexanal, heptanal, and octanal. In all cases the molecular ion (or $[\mathrm{M}-\mathrm{H}]^{-}$) was observed to be the dominant peak. The $1 \sigma$ limit of detection was estimated to be on the order of $10^{6}$ molecules in the ionization region. (J Am Soc Mass Spectrom 2004, 15, 424-430) (c) 2004 American Society for Mass Spectrometry
\end{abstract}

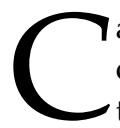
apture of low energy $(<10 \mathrm{eV})$ electrons by organic molecules has already been discussed in the literature [1]. It has been shown that the result of low energy electron attachment depends heavily upon the electron energy [1]. For instance, at a particular electron energy $(\varepsilon)$, associative attachment can occur leaving the molecular anion radical present. At a different $\varepsilon$, dissociative electron attachment can occur in which the molecule breaks apart to give a neutral radical species and a fragment anion. The favored pathway is also a function of the structural and electronic properties of the molecule. The electron capture phenomenon, known as resonance electron capture (REC), has proved to be a very useful ionization method for the detection of a variety of classes of organic compounds by mass spectrometry. It has been shown to efficiently ionize organic molecules functionalized with halogens, nitrates, sulfur, conjugated $\pi$-systems and carbonyls [2-5], as well as straight-chain hydrocarbons with minimal functionality [6]. Most recently, the versatility of REC ionization was underlined in the literature for chromatographic detection [7].

As an example of REC, the reactions at two different

Published online January 15, 2004

Address reprint requests to Dr. G. Petrucci, Department of Chemistry, University of Vermont, A218 Cook Physical Science Building, Burlington, VT 05405, USA. E-mail: Giuseppe.Petrucci@uvm.edu electron capture resonances (associative and dissociative) of nitrobenzene are shown below [8].

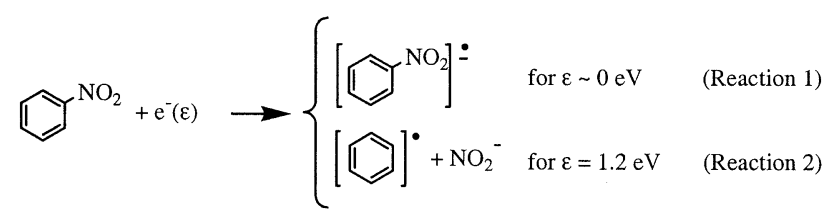

In Reaction 1, capture of zero energy electrons is an efficient process for producing intact molecular ions. In addition, through Reaction 2, higher electron energy resonances are also available for electron capture producing the $\mathrm{NO}_{2}^{-}$fragment. These higher energy resonances can improve the analytical selectivity of REC in the case of compound mixtures.

Two instruments capable of REC ionization, suited for gas phase studies, are the throchoidal electron monochromator [9] and the reversal electron attachment detector (READ) [10]. The electron monochromator uses crossed electric and magnetic fields to force different trajectories on electrons with different energies. An offset exit aperture allows monoenergetic electrons to pass into the source region of a mass spectrometer. A more detailed discussion can be found in the reference by Laramee et al. [9]. READ operates by 
reversing the direction of a focused electron beam; at the point of reversal, the kinetic energy of the electrons theoretically becomes zero. Bernius and Chutjian [10] provide a detailed description of the READ instrument. A number of other techniques, employing REC, are summarized by Chutjian et al. [1].

We are investigating a novel REC ion source for application to aerosol mass spectrometry (AMS). AMS, a central research focus in a number of laboratories, has proven to be exceptional for the analysis of single particles on line [11]. However, a limitation of existing AMS methods is the high degree of fragmentation caused during particle vaporization and ionization, particularly for organic molecules [12]. The soft ionization method reported here relies on emission of photoelectrons by the action of a tunable pulsed laser on a suitable photocathode surface. A consequence of the pulsed nature of the photoelectron emission source is a very high flux density of low energy electrons, which gives improved ionization efficiencies over existing methods, such as resonance enhanced multiphoton ionization (REMPI) [13]. The immediate need for this novel, laser-driven soft ionization source is for the analysis of single atmospheric organic particles, although there are many different areas in which such a soft, pulsed ionization source would be extremely beneficial. These include environmental analysis [14], charge transfer reactions [15], and cluster analysis [16].

Photoelectron resonance capture ionization (PERCI) operates on the principle of a laser-induced photoelectric effect to generate low energy electrons. Ionization occurs when nearby gas phase molecules capture these photoelectrons. As a first approximation, the photoelectron energy, $\varepsilon_{\mathrm{pe}}$, is equal to the difference in energy between the photons, $h \nu$, striking the metal surface and the metal work function, $\phi$.

The authors are aware of only one other recent report on the use of photoelectron attachment as an ionization mechanism for mass spectrometry [17]. Boesl et al. [17] proposed a fixed wavelength, laser-based photoelectron emission source combined with supersonic beam expansion and provided examples for its application to mass spectral analysis. The present work is intended to further evaluate the utility of low energy photoelectrons as a soft ionization source for mass spectrometry. Specifically, comparisons on the basis of sensitivity, efficiency, and selectivity are made to REMPI and electron monochromator REC. Additionally, for the first time, the tunability of PERCI is demonstrated using a tunable laser to control the photoelectron energy.

It must be emphasized that PERCI is fundamentally different from the photoelectron impact ionization (PEI) [18-20] approach also used as an ion source for mass spectrometry. PEI, in contrast to PERCI, involves the impact of high energy electrons on analyte molecules, resulting in positive ion formation, often with extensive fragmentation. While both methods rely on the photoelectric effect for generating electrons, PEI requires that the electrons be accelerated to achieve higher energies.

\section{Experimental}

A bipolar laser based TOF-MS (R. M. Jordan, Inc., Grass Valley, CA) has been adapted to PERCI analysis by inserting a $1 \mathrm{~mm}$ diameter aluminum wire $(99.9 \%$ purity from Alfa-Aesar Chemicals, Ward Hill, MA) into the ionization region of the TOF-MS. A schematic of the aerosol mass spectrometer, adapted to PERCI is given in Figure 1a. An expanded view of the PERCI source is shown in Figure 1b. A pulsed $(10 \mathrm{~Hz})$ UV laser, tunable from 235-300 nm (Opotek, Inc., Carlsbad, CA), is focused with a quartz plano-convex lens $(30 \mathrm{~cm}$ focal length) at the tip of the aluminum wire. The focused spot size on the photocathode is $0.2 \mathrm{~mm}$. Laser pulse energies are maintained between 0.5 and $1.5 \mathrm{~mJ} /$ pulse. The laser pulse $Q$ switch triggers a pulse generator (DG535 from Stanford Research Instruments, Sunnyvale, CA), which has outputs leading to the ion extraction power supply (Directed Energy, Inc., Fort Collins, CO, model PVM-4210) and the oscilloscope (LeCroy, Chestnut Ridge, NY, WavePro 7000) to time the data collection. The ion extraction grids are pulsed with $+/-$ $950 \mathrm{~V}$ at variable delay times (after the laser pulse). The extraction delay times were between 3 and $5 \mu \mathrm{s}$. The signal from the micro channel plate detector is amplified using a fast-timing preamp (Ortec, Inc., Oak Ridge, TN, model VT-120, 200x non-inverting).

Gas phase analyte was introduced using a precision leak valve (MDC Vacuum Products Corp., Hayward, CA, model ULV-150). The leak valve is not pictured in Figure 1. All solid and liquid compounds used are sufficiently volatile to vaporize under moderate vacuum with no heating necessary. The pressure of analyte in the ionization chamber is maintained between $10^{-6}$ and $10^{-5}$ torr. Instrument grade $\mathrm{SF}_{6}$ was obtained from Merriam-Graves (Charleston, NH), 2-nitrophenol was obtained from Avocado Research Chemicals, Inc. (Lancashire, UK), and all other chemicals were obtained from Aldrich Chemicals (St. Louis, MO).

\section{Results and Discussion}

The discussion that follows will be a demonstration of the PERCI technique on a number of gas phase study compounds and simple mixtures, including $\mathrm{SF}_{6}$, nitrobenzene, 2-nitrophenol, 2-pentanone, hexanal, heptanal, and octanal. All results were obtained using an aluminum photocathode. Photon wavelengths shorter than $290 \mathrm{~nm}$ are necessary to promote photoelectron emission from an aluminum photocathode $\left(\phi_{\mathrm{Al}}=4.08\right.$ $4.28 \mathrm{eV}$, depending on the condition of the metal surface). Clearly, the physical characteristics of the photocathode, such as purity, thermal and electrical conductivity, and surface condition are expected to affect the PERCI process. A stainless steel electrode, for example, was found to add significant amounts of carbon-containing fragments to the mass spectra, due to carbon present in the specific alloy used. An in depth 
a)

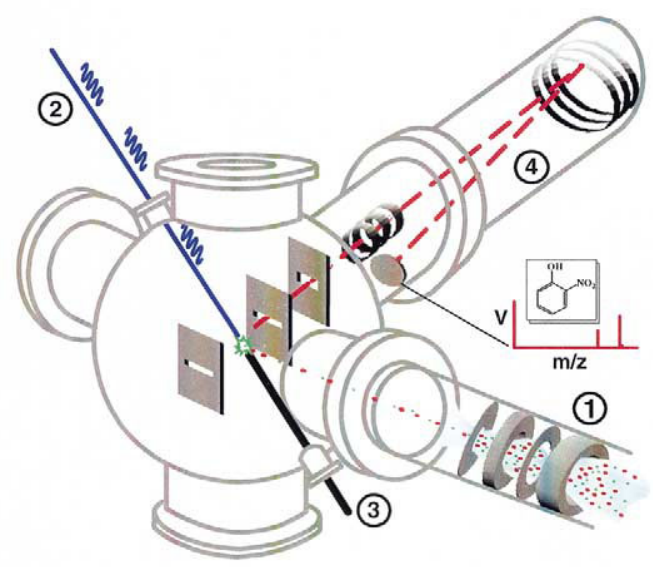

b)

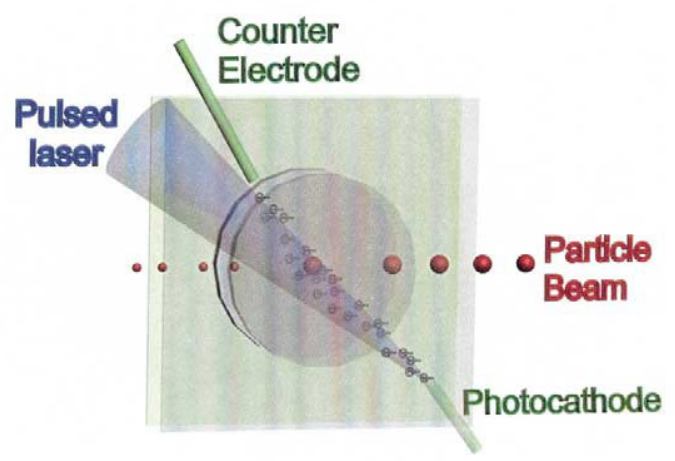

Figure 1. (a) Schematic of PERCI source adapted to aerosol mass spectrometer. There are four main components to the instrument: (1) Aerosol inlet, which is replaced with a leak valve for the current study, (2) pulsed tunable UV laser, (3) the PERCI photocathode inserted into the ion collection region of the TOF-MS, and (4) reflectron time of flight tube with micro channel plate detection, processed by a digital oscilloscope after amplification. (b) Expanded view of the PERCI source, consisting of a focused pulsed UV laser, an aluminum wire photocathode, a counter electrode (not used in the current work but will eventually be used to accelerate the electrons), and the TOF-MS extraction grids.

investigation of aluminum against other photocathode materials is presently underway in our laboratory.

\section{Demonstration of PERCI}

$\mathrm{SF}_{6}$ has an electron capture resonance at $0 \mathrm{eV}[21]$, and is used in many applications as a reference for calibrating low energy electron sources such as the electron monochromator [22]. It is used here to detect the presence of zero energy electrons formed in the PERCI source. Figure 2 is a PERCI mass spectrum of $\mathrm{SF}_{6}$ (at $\lambda$

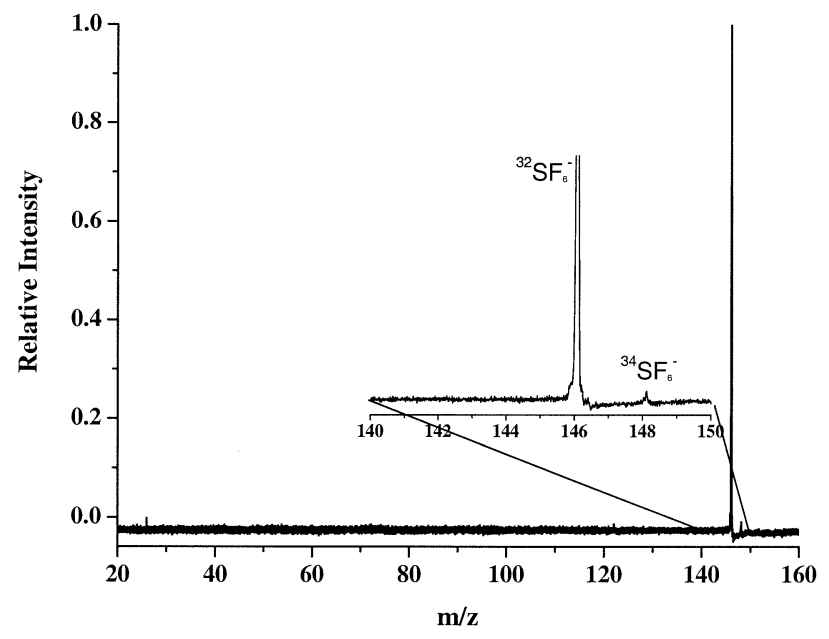

Figure 2. PERCI-TOF-MS spectrum of $\mathrm{SF}_{6}$. The spectrum is an average of 100 laser shots. $\lambda=290 \mathrm{~nm}\left(\varepsilon_{\mathrm{pe}}=0 \mathrm{eV}\right)$. The two peaks present at $\mathrm{m} / \mathrm{z} 146$ and 148 correspond to isotopes of sulfur (32 and $34 \mathrm{u})$.
$=290 \mathrm{~nm}, \varepsilon_{\mathrm{pe}}=0 \mathrm{eV}$ ) showing solely the molecular anion, $\mathrm{SF}_{6}^{-}$. This is strong evidence for the formation of high densities of zero energy electrons and their subsequent associative capture by $\mathrm{SF}_{6}$. The fragment anion, $\mathrm{SF}_{5}^{-}$, which is formed through dissociative electron capture at a photoelectron resonance energy of $0.38 \mathrm{eV}$ [21], is not detected. It is not clear whether this is a result of a smaller cross section for the dissociative capture leading to $\mathrm{SF}_{5}^{-}$, a low number density of electrons at $0.38 \mathrm{eV}$, or a combination of the two factors.

Most classes of organic compounds, including nitroaromatics [8], polyaromatics [5], and fatty acids [6], have electron capture resonances near $0 \mathrm{eV}$. PERCI can, therefore, serve as a soft and selective ionization source for a wide range of organic compounds, even those that do not absorb appreciably in the UV. As we have shown, straight chain aldehydes and ketones can also be efficiently ionized by PERCI while causing minimal molecular fragmentation. In general, the different compounds which make up these latter classes are indistinguishable using conventional laser multiphoton ionization [23]. Furthermore, these compounds do not have appreciable molar absorptivities $\left(\varepsilon_{\mathrm{m}}\right)$ in the UV and cannot be accessed efficiently by REMPI. Most aliphatic aldehydes have a weak absorption band in the wavelength range 240-360 nm due to a symmetry forbidden $\mathrm{n}-\pi^{*}$ transition [24]. For example, the maximum molar absorptivity of hexanal at $280 \mathrm{~nm}$ is only $59 \mathrm{~L} \mathrm{~mol}^{-1}$ $\mathrm{cm}^{-1}$ [25]. This is very weak in comparison with compound classes such as aromatics, which can more easily be measured by photoionization methods such as REMPI. 


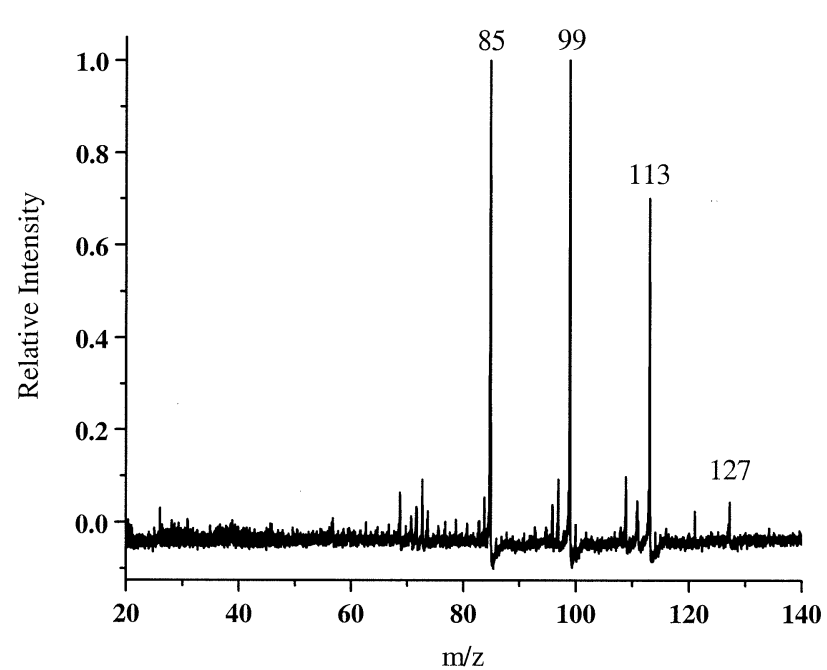

Figure 3. PERCI-TOF-MS spectrum of a mixture of 2-pentanone, hexanal, heptanal, and octanal. Spectrum is an average of 100 laser shots $(\lambda=250 \mathrm{~nm})$. Labeled peaks correspond to the $[\mathrm{M}-\mathrm{H}]^{-}$ ions of 2-pentanone (85 u), hexanal $(99 \mathrm{u})$, heptanal (113 u), and octanal (127 u).

To demonstrate the utility of PERCI to compounds that are weak absorbers of UV photons, a mixture of four compounds (hexanal, 2-pentanone, heptanal, and octanal) was analyzed by PERCI TOF-MS (Figure 3). The dominant peaks appear as the $[\mathrm{M}-\mathrm{H}]^{-}$ions at $\mathrm{m} / \mathrm{z}$ $85,99,113$, and 127 . While vapor pressures are likely a significant factor influencing the observed relative intensities of the four ions, the intensities are not exactly consistent with the relative vapor pressures (35:10:3:1, respectively). This slight discrepancy is most likely a result of differing electron attachment cross-sections and anion lifetimes for the four molecules. Investigation of the differences in the behavior of ketones versus aldehydes during PERCI analysis may be particularly interesting. Although this is only a simple mixture, this data demonstrates the potential for PERCI ionization to resolve the components of more complex non-absorbing organic mixtures.

Figure 4 shows a single shot TOF mass spectra of hexanal, in which the $[\mathrm{M}-\mathrm{H}]^{-}$ion is the dominant mass peak. Typical signal to noise values for single shot mass spectra range from 10-100, varying with laser power density, at gas phase pressures of $10^{-5}$ torr. In Figure 4, for example, the signal-to-noise on the hexanal peak at $m / z=99$ for a single laser shot is 60 . The hexanal vapor pressure is $1 \times 10^{-5}$ torr, corresponding to a molecular density of $3 \times 10^{11}$ molecules $\mathrm{cm}^{-3}$. Ions are formed in a volume given approximately by the area of the laser spot on the photocathode $\left(0.12 \mathrm{~mm}^{2}\right)$ and length of the ion collection region $(3 \mathrm{~mm})$. For our experimental conditions, the ionization volume is $1 \times$ $10^{-4} \mathrm{~cm}^{3}$, containing $1 \times 10^{8}$ hexanal molecules. Assuming the molecules are perfectly coupled to the ionization region, the corresponding $1 \sigma$ limit of detection is estimated to be $2 \times 10^{6}$ molecules.

The sensitivities of PERCI versus REMPI $(1+1)$ [13]

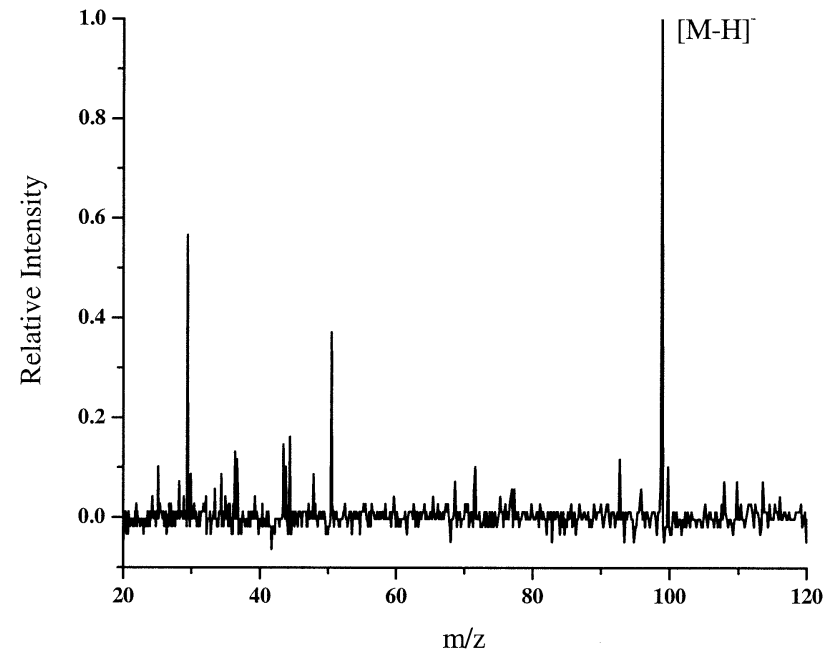

Figure 4. Single-shot PERCI-TOF-MS spectrum of hexanal. $\lambda=$ $280 \mathrm{~nm}\left(\varepsilon_{\mathrm{pe}}=0.1_{5} \mathrm{eV}\right)$.

are compared qualitatively in the simultaneous positive and negative ion spectra of nitrobenzene (Figure $5 \mathrm{a}$ and $\mathrm{b}$, respectively). The two spectra were taken by making use of the bipolar TOF-MS. REMPI results in positive ion formation, while PERCI results in the formation of negative ions. The wavelength of the laser was set at 249 $\mathrm{nm}\left(4.9_{8} \mathrm{eV}\right)$ so that simultaneous absorption of two photons would ionize the nitrobenzene molecules (ionization energy $=9.9_{4} \mathrm{eV}, 1+1$ REMPI scheme). Under these conditions, PERCI proceeds via dissociative electron attachment $\left(\varepsilon_{\mathrm{ph}}=0.7_{0} \mathrm{eV}\right)$ resulting in predominantly the $\mathrm{NO}_{2}^{-}$fragment anion. As seen in Figure 5a and $b$, under identical experimental conditions, PERCI ionization occurs readily while the same laser energy density is insufficient for REMPI, demonstrating the

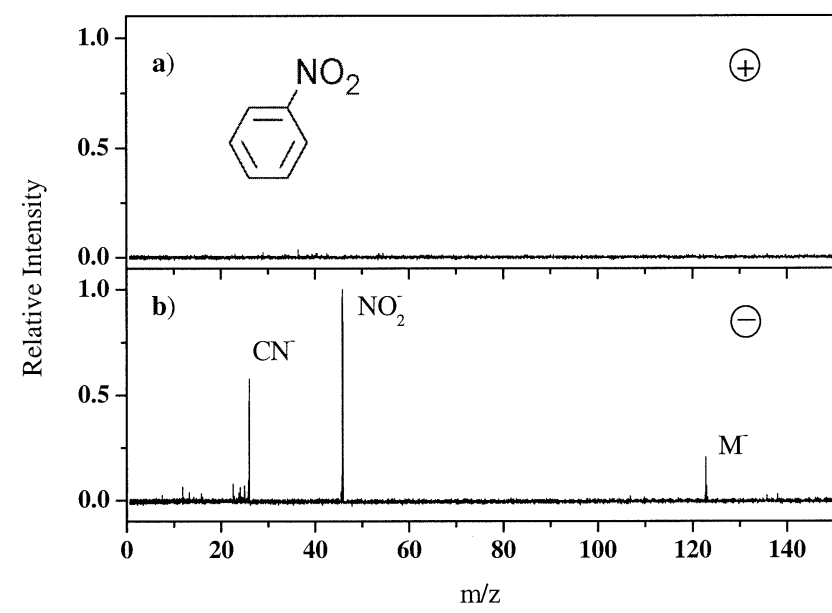

Figure 5. Simultaneous (a) positive and (b) negative ion analysis of nitrobenzene using bipolar TOF-MS. Spectra obtained from a single laser shot. $\lambda=249 \mathrm{~nm}\left(\varepsilon_{\mathrm{pe}}=0.7_{0} \mathrm{eV}\right)$. The two spectra compare REMPI (positive ions) and PERCI (negative ions) at an identical laser energy density. Laser wavelength was not tuned to an electron capture resonance of $\mathrm{M}^{-}$, so $\mathrm{NO}_{2}^{-}$is dominant peak (see text). 


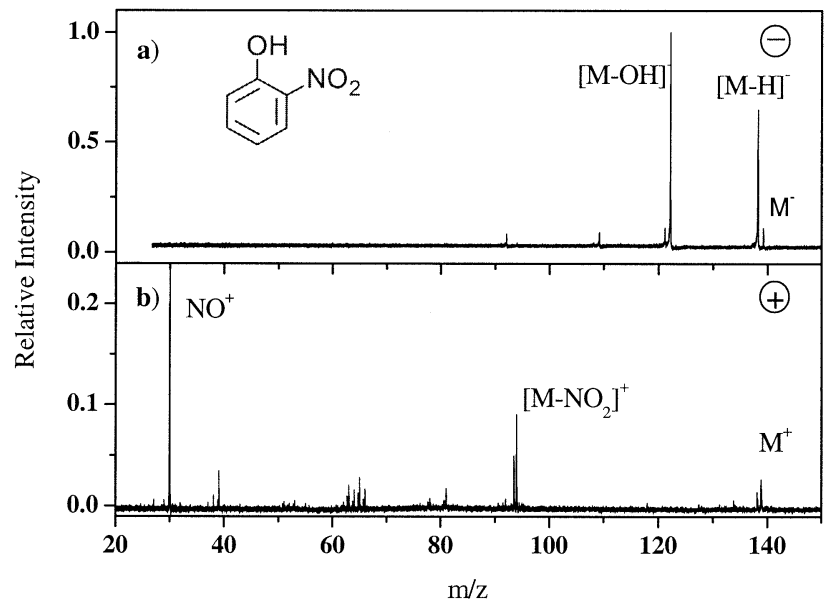

Figure 6. Comparison of (a) PERCI and (b) REMPI fragmentation patterns for 2-nitrophenol. PERCI results in $\left[\mathrm{M}-\mathrm{H}^{-}\right.$and [M $-\mathrm{OH}]^{-}$peaks while REMPI results in primarily $\mathrm{NO}^{+}$(note that this peak is normalized to an intensity of 1). Spectra are averages of 100 laser shots $(\lambda=260 \mathrm{~nm})$.

enhanced sensitivity achieved by PERCI. It is important to note that at the wavelength used ( $249 \mathrm{~nm})$, nitrobenzene exhibits an absorbance maximum $\left(\varepsilon_{\mathrm{m}}=2510 \mathrm{~L}\right.$ $\mathrm{mol}^{-1} \mathrm{~cm}^{-1}$ [25]), which should result in an improved REMPI signal.

The comparison of PERCI (Figure 6a) and REMPI (Figure 6b) mass spectra of 2-nitrophenol provides an example of the soft ionization afforded by PERCI. These mass spectra were not taken simultaneously, as in the previous example. The laser energy was increased in order to produce a measurable REMPI mass spectrum. The wavelength of the laser was $260 \mathrm{~nm}$, sufficient to ionize 2-nitrophenol via the $(1+1)$ REMPI mechanism. 2-nitrophenol has an absorbance maximum at $260 \mathrm{~nm}$ $\left(\varepsilon_{\mathrm{m}}=3980 \mathrm{~L} \mathrm{~mol}^{-1} \mathrm{~cm}^{-1}\right)$ which should result in an enhanced REMPI signal. While REMPI produces predominantly the $\mathrm{NO}^{+}$ion (note that the spectrum has been expanded to show the smaller carbon fragments and that the $\mathrm{NO}^{+}$peak has a normalized intensity of $1.00)$, which could potentially come from any number of unknown nitro-containing organics, PERCI produces only two high mass fragments, the $[\mathrm{M}-\mathrm{H}]^{-}$and $[\mathrm{M}-$ $\mathrm{OH}]^{-}$ions. Both of these ions closely resemble the parent molecule, greatly simplifying identification.

It is relevant here to compare PERCI with electron monochromator sources used for electron capture studies. While no direct comparison could be made experimentally between the PERCI source and the electron monochromator, electron current may be used as a suitable gauge for the relative sensitivities of the two techniques. Aluminum has a photoelectron quantum efficiency, defined as the ratio of photoelectrons emitted to the number of incident photons, of about $10^{-4}$ [26]. Therefore, a $1 \mathrm{~mJ}$ pulsed laser, with a $10 \mathrm{~ns}$ pulse duration, operating at $290 \mathrm{~nm}$ and $10 \mathrm{~Hz}$ will yield a continuous current of about $200 \mathrm{nA}$. This is comparable to current values reported for electron monochroma- tors, ranging from $0.5-2 \mu \mathrm{A}$ [9]. However, due to the pulsed nature of the PERCI source, its sensitivity is enhanced since the instantaneously high electron current is more effectively coupled to the pulsed ion collection of the TOF-MS. In evaluating the sensitivity of PERCI, therefore, it is more useful to consider the instantaneous current during the $10 \mathrm{~ns}$ laser pulse, which can theoretically reach values of 10-100A. In preliminary experiments, instantaneous photocurrents as high as $10 \mathrm{~mA}$ have been measured. The difference between the expected maximum instantaneous current $(10-100 \mathrm{~A})$ and that measured $(10 \mathrm{~mA})$ may be explained in part by space charge effects near the cathode surface [27]. Nonetheless, the measured instantaneous photocurrent is several orders of magnitude greater than that possible with electron monochromators. Experiments are underway to determine the absolute photoelectron current of the PERCI source.

Easy tunability of $\varepsilon_{\mathrm{pe}}$ is also a principal feature of the PERCI ion source. Tunable photoelectron energies could be used to great analytical advantage, providing capabilities approaching isomeric selectivity. For example, of the two isomers $p-\mathrm{NO}_{2}$ and $m-\mathrm{NO}_{2}$ azobenzene, only the $p-\mathrm{NO}_{2}$ isomer has an electron capture resonance at $0.4 \mathrm{eV} \mathrm{[29]} \mathrm{leading} \mathrm{to} \mathrm{formation} \mathrm{of} \mathrm{the} \mathrm{molec-}$ ular anion. Therefore, in an isomeric mixture, tuning the photon wavelength to $264.9_{4} \mathrm{~nm}$ (for an aluminum photocathode with $\phi=4.2_{8} \mathrm{eV}$ ) will produce the $\mathrm{M}^{-}$ ion of the $p-\mathrm{NO}_{2}$ isomer only. This is a powerful advantage over conventional ionization approaches which cannot easily resolve organic isomers, especially if significant molecular fragmentation is caused.

The photoelectron energy may be tuned in two ways: application of an auxiliary electric field and/or tuning of the photon energy. The additional force of an applied potential, $\mathrm{V}_{\alpha}$, will accelerate the photoelectrons, increasing $\varepsilon_{\mathrm{pe}}$ accordingly. The magnitude of $\varepsilon_{\mathrm{pe}}$, then, is in actuality a function of $\mathrm{V}_{\alpha}$, as well as $\phi$ and $\mathrm{h} \nu$ :

$$
\varepsilon_{p e}=h \nu-\phi+V_{\alpha}
$$

While $\mathrm{h} \nu$ is an experimentally controllable parameter, the presence of large electron densities over a small area and the remaining image potential on the surface of the photocathode have both been shown to contribute significantly to $\mathrm{V}_{\alpha}$ under certain conditions [27]. Space charge effects are, therefore, expected to influence the actual energy of the photoelectrons, leading to broadening of the photoelectron energy distribution. Experimental control of $\mathrm{V}_{\alpha}$ and therefore $\varepsilon_{\mathrm{pe}}$ is not straightforward as a result. Nonetheless, initial results with nitrobenzene show that tuning of $\varepsilon_{\mathrm{pe}}$ is possible by tuning the laser wavelength.

The nitrobenzene spectrum in Figure $5 \mathrm{~b}$ was taken at a photoelectron energy of $0.7_{0} \mathrm{eV}$. Reaction 2 is clearly the dominant pathway at this photon energy, leading to the $\mathrm{NO}_{2}^{-}$fragment ion. It was found that the molecular ion could be maximized at photon energies closer to the 


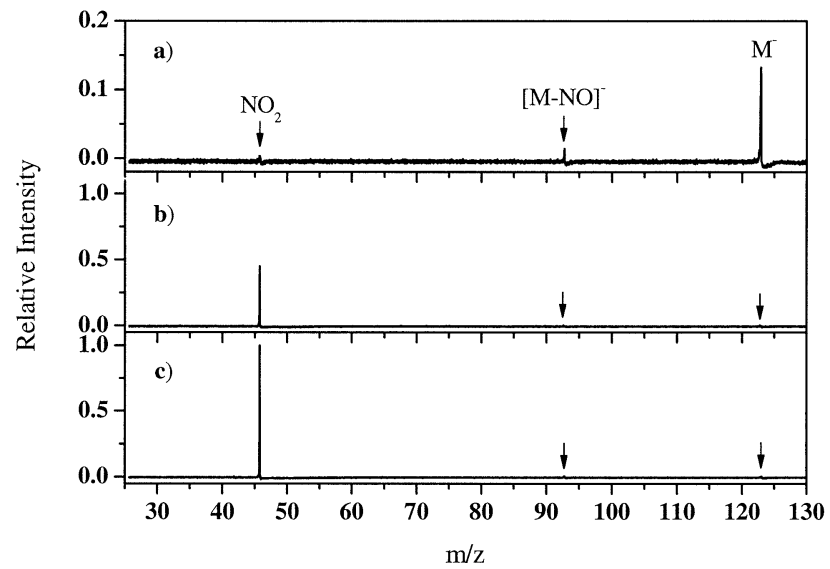

Figure 7. Mass spectra of nitrobenzene taken at photon energies of (a) $4.28 \mathrm{eV}(290 \mathrm{~nm}),(\mathbf{b}) 4.77 \mathrm{eV}(260 \mathrm{~nm})$, and (c) $5.18 \mathrm{eV}(240$ $\mathrm{nm}$ ), corresponding to $\varepsilon_{\mathrm{pe}}=0,0.49$ and $0.90 \mathrm{eV}$, respectively. Spectra averages of 100 laser shots have been normalized for photon flux density (photons $\mathrm{s}^{-1} \mathrm{~cm}^{-2}$ ) at the photocathode. All signals in the three spectra are normalized to the $\mathrm{NO}_{2}^{-}$peak in spectrum (c).

work function of aluminum $(\lambda \sim 290 \mathrm{~nm})$, where associative photoelectron attachment (Reaction 1) is favored. Figure 7 demonstrates this further, showing mass spectra of nitrobenzene at three different photon energies $4.2_{8}(290 \mathrm{~nm}), 4.7_{7}(260 \mathrm{~nm})$, and $5.1_{8} \mathrm{eV}(240$ $\mathrm{nm})$. Taking $\phi_{\mathrm{Al}}=4.2_{8} \mathrm{eV}$, the photoelectron energies are expected to be $0,0.5$ and $0.9 \mathrm{eV}$, respectively. The $\left[\mathrm{M}^{-}\right] /\left[\mathrm{NO}_{2}^{-}\right]$ratios are $39,1.4 \times 10^{-2}$, and $5.6 \times 10^{-3}$ in Figure $7 \mathrm{a}, \mathrm{b}$, and $\mathrm{c}$, respectively. These values are consistent with a previous report on the REC behavior of nitrobenzene [8] where $\mathrm{M}^{-}$was found to dominate at $\varepsilon_{\mathrm{pe}}=0 \mathrm{eV}$, while the $\mathrm{NO}_{2}^{-}$resonance appears near $\varepsilon_{\mathrm{pe}}$ $=0.5 \mathrm{eV}$ and continues to rise, reaching its peak resonance at $\varepsilon_{\mathrm{pe}}=1.2 \mathrm{eV}$. The intensity of the $\mathrm{NO}_{2}^{-}$ion in Figure $7 \mathrm{~b}$ and $\mathrm{c}$ is significantly higher than that of the $\mathrm{M}^{-}$ion in Figure $7 \mathrm{a}$, which is expected since the reported cross section for the formation of $\mathrm{NO}_{2}^{-}$is roughly twice that for the formation of the molecular ion [8].

In the present study, the exact electron energies and bandwidths are not accurately known, rendering a quantitative comparison of the relative peak heights to capture cross sections reported in the literature difficult. The trends, however, show that the relationship between photon energy and photoelectron energy is consistent with theory and that the selectivity provided by REC ionization can be realized with the tunable PERCI source. Work is underway to investigate, more closely, these results and the effect of laser wavelength on photoelectron energies and energy distributions. Additionally, it will be advantageous to have an experimentally controllable $\mathrm{V}_{\alpha}$ in order to access $\varepsilon_{\mathrm{pe}}$ values in the range of $6-8 \mathrm{eV}$, where many polyaromatics exhibit an $[\mathrm{M}-\mathrm{H}]^{-}$resonance [5].

\section{Future Research Aims}

PERCI as an ion source for time-of-flight mass spectrometry is still very much in its infancy. As such, there is a great deal of work yet to be done to better understand the fundamental aspects of photoelectron generation and ion formation. For example, it is presently not clear where the photoelectron capture takes place relative to the photocathode surface. A detailed spatial characterization will be especially important in applying PERCI toward the real-time analysis of aerosols, which must be introduced to the instrument as a tightly focused particle beam [28]. Overlap of the particle beam and the photoelectron cloud is essential for efficient ionization of the vaporized particles. There is also uncertainty as to whether the electron capture occurs instantaneously or after some delay. It has been observed that the mass spectrum for a given molecule is dependant upon the delayed pulse extraction time, that is, the time between the ionizing laser pulse and the ion extraction pulse. The reason behind this is currently being explored. Additionally, the tunability of the electron energy and distribution of photoelectron energies need to be investigated further. We believe this capability has great potential for the resolution of naturally occurring mixtures of organics. Clearly, a thorough understanding of the underlying processes involved in PERCI is essential to improving the ionization efficiency and selectivity further.

\section{Conclusions}

PERCI is a promising method for the generation of negative ions with minimal molecular fragmentation and high sensitivity. As proof-of-principle, high fluxes of near-zero energy electrons have been generated, leading to the soft ionization of two classes of organic molecules: nitro-aromatics and straight-chain carbonyls. The potential exists for PERCI to resolve complex mixtures of organics. In comparison to REMPI, conventionally used as a soft and efficient ion source in aerosol mass spectrometry, PERCI offers a softer, more sensitive ionization of organic molecules in a broader range of compound classes, including those that are not accessible by REMPI due to weak absorption probabilities in the UV. By virtue of its pulsed nature, PERCI is estimated to be more sensitive than the electron monochromator ion source when coupled to a pulsed mass analysis method such as TOF-MS. Assuming complete coupling of the analyte to the ionization region, the limit of detection is estimated to be on the order of $10^{6}$ molecules, corresponding roughly to the detection of a pure organic particles in the ultrafine region (diameter $<200 \mathrm{~nm}$ ). It was demonstrated for the first time that tuning of the ionization source could be accomplished by tuning the photoelectron energy via laser wavelength. In this manner, ready control of the photoelectron energy to promote formation of molecular anions or selected fragments could be used effectively to 
simplify mass spectra from complex mixtures of organic compounds and greatly enhance identification capabilities. Further investigation into the fundamental processes of PERCI will undoubtedly lead to the increased versatility of this novel ionization technique.

\section{Acknowledgments}

The authors gratefully acknowledge the financial support of the American Society for Mass Spectrometry, through a 2003 research award, and the National Center for Environmental Research (NCER) STAR Program, EPA (Fellowship No. 91615301 - 0).

\section{References}

1. Chutjian, A.; Garscadden, A.; Wadehra, J. M. Electron Attachment to Molecules at Low Electron Energies. Phys. Rep. 1996, 264, 393-470.

2. Imashev, U. B.; Khvostenko, O. G.; Lukin, V. G.; Shereshovets, V. G.; Mazunov, V. A. Resonance Electron Capture NegativeIon Mass Spectrometry for Analysis of Petroleum and Petroleum Products: Thiophene S-Oxides. J. Anal. Chem. 1998, 53, 578-582.

3. Imashev, U. B.; Khvostenko, O. G.; Lukin, V. G.; Vezirov, R. R.; Khairudinov, I. R.; Tuktarov, R. F.; Mazunov, V. A. Resonance Electron Capture Negative-Ion Mass Spectrometry for Analysis of Petroleum and Petroleum Products: Determination of Polysulfides. J. Anal. Chem. 1998, 53, 779-783.

4. Wei, J.; Liu, S.; Fedoreyev, S. A.; Voinov, V. G. A Study of Resonance Electron Capture Ionization on a Quadrupole Tandem Mass Spectrometer. Rapid Commun. Mass Spectrom. 2000, 14, 1689-1694.

5. Tobita, S.; Meinke, M.; Illenberger, E.; Christophorou, L. G.; Baumgartel, H. Polycyclic Aromatic Hydrocarbons: Negative Ion Formation Following Low Energy $(0-15 \mathrm{eV})$ Electron Impact. Chem. Phys. 1992, 161, 501-508.

6. Voinov, V. G.; Boguslavskiy, V. M.; Elkin, Y. N. Resonance Electron Capture for Determining Double Bond and Hydroxy Group Locations in Fatty Acids. Org. Mass Spectrom. 1994, 29, 641-646.

7. Voinov, V. G.; Vasil'ev, Y. V.; Morre, J.; Barofsky, D. F.; Deinzer, M. L.; Gonin, M.; Egan, T. F.; Fuhrer, K. A Resonant Electron Capture Time-of-Flight MS with Trochoidal Electron Monochromator. Anal. Chem. 2003, 75, 3001-3009.

8. Modelli, A.; Venuti, M. Empty Level Structure and Dissociative Electron Attachment in Gas-Phase Nitro Derivatives. Int. J. Mass Spectrom. 2001, 205, 7-16.

9. Laramée, J. A.; Mazurkiewicz, P.; Berkout, V.; Deinzer, M. L. Electron Monochromators-Mass Spectrometer Instrument for Negative Ion Aanalysis of Electronegative Compounds. Mass Spectrom. Rev. 1996, 15, 15-42.

10. Bernius, M. T.; Chutjian, A. Application of Reversal Electron Attachment for Ultra Sensitive Detection of Thermal ElectronAttaching Molecules: $\mathrm{CCl}_{4}$ and $\mathrm{C}_{6} \mathrm{H}_{5} \mathrm{NO}_{2}$. Anal. Chem. 1990, 62, 1345-1349.

11. Noble, C. A.; Prather, K. A. Real-Time Single Particle Mass Spectrometry: A Historical Overview of a Quarter Century of the Chemical Analysis of Aerosols. Mass Spectrom. Rev. 2000, 19, 248-274.

12. Hunt, A. L.; Petrucci, G. A. Analysis of Ultra Fine and Organic Particles by Aerosol Mass Spectrometry. Trac-Trends in Analytical Chemistry. 2002, 21, 74-81.

13. Boesl, U.; Weinkauf, R.; Weickhardt, C.; Schlag, E. W. Laser Ion Sources for Time-of-Flight Mass Spectrometry. Int. J. Mass Spectrom. Ion Processes 1994, 131, 87-124.

14. Ong, V. S.; Hites, R. A. Electron Capture Mass Spectrometry of Environmental Contaminants. Mass Spectrom. Rev. 1994, 13, 259-283.

15. Chattoraj, M.; Laursen, S. L.; Paulson, B.; Chung, D. D.; Closs, G. L.; Levy, D. H. Intramolecular Electron Transfer in a Molecular Beam. J. Chem. Phys. 1992, 96, 8778-8784.

16. Mark, T. D. Free Electron Attachment to van der Waals Clusters. Int. J. Mass Spectrom. Ion Processes 1991, 107, 143-164.

17. Boesl, U.; Bassmann, C.; Drechsler, G.; Distelrath, V. LaserBased Ion Source for Mass Spectrometry and Laser Spectroscopy of Negative Ions. Eur. Mass Spectrom. 1999, 5, 455-470.

18. Schriemer, D. C.; Li, L. Laser-Induced Surface Ionization in a Time-of-Flight Mass Spectrometer. Rev. Sci. Instrum. 1995, 66, $55-62$.

19. Colby, S. M.; Reilly, J. P. Photoemission Electron Impact Ionization in Time-of-Flight Mass Spectrometry: An Examination of Experimental Consequences. Int. J. Mass Spectrom. Ion Processes 1994, 131, 125-138.

20. Wang, L.; Li, H. Y.; Bai, J. L.; Hua, X. Q.; Lu, R. C. Application of Laser Induced Photoemission Electron in Time-of-Flight Mass Spectrometry. Int. J. Mass Spectrom. 1998, 181, 43-50.

21. Christophorou, L. G.; Olthoff, J. K. Electron Attachment Cross Sections and Negative Ion States of $\mathrm{SF}_{6}$. Int. J. Mass Spectrom. 2001, 205, 27-41.

22. Mazunov, V. A.; Vasil'ev, Y. V.; Muftakhov, M. V. Reference for Energy-Scale Calibration in Mass Spectrometry of Resonance Electron Capture. Instrum. Experim. Tech. 1991, 34, 378-380.

23. Silva, P. J.; Prather, K. A. Interpretation of Mass Spectra from Organic Compounds in Aerosol Time-of-Flight Mass Spectrometry. Anal. Chem. 2000, 72, 3553-3562.

24. Calvert, J. G.; Pitts, J. N. J. Photochemistry; John Wiley: New York, 1966, pp 372-375.

25. The Sadtler Handbook of Ultraviolet Spectra; Simons, W. W., Ed.; Sadtler Research Laboratories: Philadelphia, PA; 1979.

26. Kawamura, K.; Toyoda, K.; Kawai, M. Generation of Relativistic Photoelectrons Induced by Excimer Laser Irradiation. Appl. Phys. Lett. 1984, 45, 307-309.

27. Gilton, T. L.; Cowin, J. P.; Kubiak, G. D.; Hamza, A. V. Intense Surface Photoemission: Space Charge Effects and Self-Acceleration. J. Appl. Phys. 1990, 68, 4802-4810.

28. Petrucci, G. A.; Farnsworth, P. B.; Cavalli, P.; Omenetto, N. A Differentially Pumped Particle Inlet for Sampling of Atmospheric Aerosols into a Time-of-Flight Mass Spectrometer: Optical Characterization of the Particle Beam. Aerosol Sci. Technol. 2000, 33, 105-121.

29. Vasil'ev, Y. V.; Mazunov, V. A.; Nazirov, E. R. Resonance Electron-Capture Mass Spectra of Azobenzenes and its Monosubstituted Derivatives. Org. Mass Spectrom. 1991, 26, 739-741. 